\title{
Le financement des écoles publiques à Montréal et à Toronto (1841-1997) : un baromètre pour mesurer les rapports entre la majorité et la minorité
}

\author{
Jean-Philippe Croteau
}

\section{RÉSUMÉ}

Cette étude propose d'examiner les modes de financement scolaire à Montréal et à Toronto, entre 1841 et 1997, sous l'angle sociopolitique d'un baromètre pour mesurer les rapports sociaux entre la majorité et la minorité. Il ressort de nos recherches que l'État provincial ontarien accorde, avant les années 1960, la primauté aux écoles publiques qui disposent à la fois de la reconnaissance politique et de l'accès aux ressources financières, tandis que les écoles séparées sont soumises à des contraintes légales et financières qui les confinent à un espace social dévolu à la minorité. À l'opposé, le gouvernement québécois reconnaît l'égalité et l'autonomie des acteurs catholiques et protestants, tant sur les plans administratif, pédagogique que financier. Ainsi, il apparaît que la scène scolaire constitue un espace névralgique de discussions, de négociations et même d'affrontements entre les différents groupes religieux et culturels pour faire valoir leurs conceptions éducatives et obtenir l'accès aux ressources financières.

\section{ABSTRACT}

This study proposes to examine the ways in which schools were financed, both in Montreal and Toronto, between 1841 and 1997, from the perspective of a socio-political barometer measuring the social relationships between majority and minority. It is clear from our research that prior to the 1960s the Ontario provincial government gave priority to public schools, providing both political recognition and access to financial resources, while separate schools were subjected to financial and legal constraints and confined to the social space vested to the minority. In contrast, the Quebec government recognized the equality and autonomy of actors both Catholics and Protestants on administrative, educational, and financial levels. Thus, the educational scene appears to be a space that is sensitive to discussions, negotiations, and even clashes between different religious and cultural groups, where each can promote their conceptions of education and gain access to financial resources. 
À l'aube de la Confédération, les représentants de la minorité protestante au CanadaEst partent en campagne pour convaincre les décideurs politiques de leur accorder des garanties constitutionnelles pour protéger leur système scolaire et assurer son autonomie vis-à-vis de la majorité catholique et francophone. Ces revendications scolaires trouvent écho auprès de la minorité catholique du Canada-Ouest qui lui emboîte le pas et réclame la parité scolaire avec les protestants du Canada-Est. Si les deux minorités obtiennent une protection constitutionnelle pour leurs écoles en vertu de l'article 93 de l'Acte de l'Amérique du Nord britannique (AANB), les dirigeants politiques québécois et ontariens interpréteront différemment l'esprit de la constitution lorsqu’ils légiféreront en matière scolaire.

Force est de constater que l'histoire du système scolaire au Québec et en Ontario suit une trajectoire inversée et même une inversion des rôles entre les secteurs éducatifs de la majorité et de la minorité dans ces deux provinces. La compréhension de ce phénomène demeure indissociable du régime de financement scolaire, mis en place à partir de la seconde moitié du XIX ${ }^{\mathrm{e}}$ siècle, qui définit les conditions d'existence et surtout d'expansion des systèmes scolaires au Québec et en Ontario et reproduit en même temps les rapports de force entre les groupes culturels et religieux. Ainsi, au cours de la période étudiée, le gouvernement québécois souscrit à une vision dualiste des rapports ethnoreligieux qui reconnaît l'égalité et l'autonomie des acteurs catholiques et protestants tant sur les plans administratif, pédagogique que financier. À l'opposé, l'État provincial ontarien obéit à une logique majoritaire qui reflète la volonté du groupe prépondérant, les protestants, en accordant la primauté aux écoles publiques qui disposent à la fois de la reconnaissance politique et de l'accès aux ressources financières, tandis que les écoles séparées se voient désigner un espace éducatif dévolu à la minorité catholique et délimité par des contraintes légales et financières. Ces concordats scolaires, signés entre l'État, la majorité et la minorité religieuse, connaîtront peu de changements avant les années 1960, alors que l'État change de vocation pour assurer l'égalité des chances et la justice sociale à ses citoyens en amorçant des réformes destinées à démocratiser le système d'éducation.

Aux fins de démonstration, nous avons examiné le cas des commissions scolaires des villes de Montréal et de Toronto afin d'assurer une cohérence au cadre d'analyse et observer des données comparables. Bien qu'elles aient fait rarement l'objet d'études comparatives, Montréal et Toronto disposent d'un cadre sociologique qui comporte certaines similitudes. En effet, les deux cités ont connu un essor, à partir de la deuxième moitié du XIX ${ }^{\mathrm{e}}$ siècle, caractérisé par les phénomènes de l'urbanisation, de l'industrialisation et de l'immigration. Dans ce contexte, les commissions scolaires montréalaises et torontoises ont composé avec les défis que posent une nouvelle économie industrielle, les transformations des rapports sociaux et la gestion de la diversité culturelle et religieuse. De plus, il est important de rappeler que les commissions scolaires montréalaises disposent, contrairement à celles de Toronto, d'un régime financier différent du reste de la province, ce qui rendait impossible la comparaison du Québec et de l'Ontario sans tenir compte du statut particulier de Montréal.

Il faut préciser d'emblée que nous n'entrons pas dans un champ complètement inexploré. Toutefois, les recherches sur la question du financement des écoles 
publiques à Montréal et à Toronto ont surtout visé jusqu'à ce jour à évaluer la qualité et la variété des services éducatifs offerts par les commissions scolaires et leur impact sur la scolarisation de la population. Or, peu d'études ont abordé les modes de financement scolaire sous l'angle sociopolitique d'un baromètre pour mesurer les relations entre la majorité et la minorité. Au-delà de l'exercice comptable, la comparaison des modes de financement scolaire à Montréal et à Toronto comporte un potentiel important pour comprendre les relations entre la majorité et les minorités au sein du champ scolaire. Cette étude ne prétend pas établir un bilan définitif compte tenu de la longueur de la période examinée qui nous restreint sur le plan de l'analyse. Elle vise plutôt à proposer des interprétations sur la question des relations entre la majorité et les minorités au sein de la sphère éducative en brossant un panorama général du mode de financement scolaire à Montréal et à Toronto. Pour ce faire, nous avons consulté essentiellement les rapports annuels et les procès-verbaux des commissions scolaires montréalaises et torontoises, les éditoriaux de la presse, les mémoires soumis par les acteurs éducatifs aux commissions d'enquête et au gouvernement, ainsi qu'un nombre important d'études rédigées par les historiens sur la question.

\section{Les écoles séparées en Ontario, une guerre des religions?}

En Ontario, les écoles publiques fondées dans les années 1840 et 1850, constituent une réponse formulée par les élites politiques et sociales de l'époque aux crises politiques des années 1830 et aux bouleversements sociaux engendrés par l'industrialisation. Ces élites, descendantes des loyalistes qui ont fui la Révolution américaine, valorisent une école publique qui préserve la société des risques de contagion républicaine, de la dilution des valeurs religieuses et spirituelles et de l'éclatement des structures sociales causé par les insurrections politiques et l'industrialisation. À partir de 1844, Egerton Ryerson devient le premier surintendant de l'éducation du CanadaOuest et il restera en poste jusqu'en 1876. Bien que Ryerson ne soit pas le fondateur des écoles publiques de l'Ontario, il en reste le principal architecte.

Ryerson demeure un homme de son époque, préoccupé par les bouleversements sociaux et politiques qui caractérisent le Haut-Canada dans la première moitié du XIX ${ }^{\mathrm{e}}$ siècle. Il prône l'intervention de l'État en éducation pour encadrer les masses populaires et remédier aux maux qui menacent l'ordre social. ${ }^{1}$ À cet effet, l'éducation pour Ryerson doit être chrétienne, patriotique, gratuite et obligatoire. Dès les années 1840, avant même d'occuper une position officielle dans l'administration scolaire, il définissait ainsi les visées de l'éducation :

The object of education, rightly understood is, first, to make youth good men - good members of universal society; secondly, to fit them for usefulness to that particular society of which they constitute an integral part — to form their principles and habits - to develop their talents and dispositions in such a way, as will be most serviceable to the institutions under which they live [...]. ${ }^{2}$ 
et de socialisation engagée dans la sanctification de l'individu et de la rédemption politique, économique et spirituelle de la société. ${ }^{3}$ Inspirée des principes moraux des Saintes Écritures sur lesquels repose l'ordre politique, social et culturel, l'éducation s'adresse à l'ensemble des individus indépendamment de leur classe sociale, de leur appartenance religieuse et de leur origine ethnique. Elle vise à renforcer la cohésion sociale, à faire reculer les vices moraux et les maux sociaux menacés par l'ignorance et à former des citoyens éclairés pour protéger la société et les institutions démocratiques naissantes contre les esprits de parti et les démagogues. ${ }^{4}$ Cependant, la vision de Ryerson ne fait pas l'unanimité. Dès la seconde moitié du XIX ${ }^{\mathrm{e}}$ siècle, l'épiscopat catholique, dirigé par nouvel évêque de Toronto, M ${ }^{\mathrm{gr}}$ Armand-François-Marie de Charbonnel, ultramontain convaincu, conteste les visées éducatives de Ryerson et réclame que les catholiques possèdent leurs propres écoles.

La question scolaire au Canada-Ouest s'inscrit dans la foulée des luttes séculaires menées par les Églises protestantes contre les prétentions du pape à subordonner le pouvoir politique à son autorité spirituelle et aux traditions de l'Église catholique. L'opposition du pape au libéralisme politique a nourri une forme de nationalisme protestant, incarné entre autres par le mouvement évangélique qui cherche à réaliser les desseins de Dieu en restaurant les valeurs chrétiennes — voire protestantes — dans une société menacée par la déchristianisation engendrée par le matérialisme et le "papisme».

L'arrivée des milliers d'immigrants fuyant la Grande Famine en Irlande, à partir de la fin des années 1840, fait éclater le consensus d'une société qui, particulièrement à Toronto, se percevait comme un bastion britannique et protestant. ${ }^{5}$ Les irlandais catholiques sont considérés par la majorité protestante comme un groupe porteur de nombreux problèmes sociaux (pauvreté, chômage, criminalité, etc.) et qui constituent une menace pour l'ordre public. Les conflits entre protestants et catholiques constitueront un élément central de la vie sociale torontoise dans la deuxième moitié du XIX ${ }^{e}$ siècle, conflits qui vaudront à la métropole du Canada-Ouest le surnom de Belfast of the North.

Enfin, dans les années 1840 et 1850, à la suite de l'Union du Haut-Canada et du Bas-Canada, beaucoup de protestants craignent que les Canadiens français, en faisant alliance avec les Irlandais, se mettent au service des ambitions politiques du pape et tentent d'instaurer une French Domination. Dans ce contexte, la question des écoles catholiques - ou séparées — revêt une question hautement politique. Les protestants défendent toute altération du caractère britannique et protestant de la société et entendent bien refuser des concessions aux catholiques dans le champ scolaire ou politique. Les écoles séparées en Ontario apparaissent donc pour la majorité protestante comme une porte ouverte à l'ingérence papale qui menace les libertés civiles, encourage le sectarisme religieux et affaiblit l'unité nationale. Cette opinion est confortée par les manifestations ultramontaines d'une Église catholique qui grandit en influence au Canada-Est. ${ }^{6}$

C'est dans ce contexte que, dans les années 1850, l'épiscopat catholique, les sociétés nationalistes et la presse irlandaise mènent une croisade pour obtenir la reconnaissance d'un statut officiel pour les écoles catholiques. Pour l'épiscopat catholique de Toronto, 
l'école publique constitue un instrument d'assimilation et de prosélytisme aux mains de la majorité protestante. L'école publique, en dépit de son caractère prétendument non confessionnel, est vue comme une école protestante déguisée qui conduirait le catholique à la perte de sa foi. En fait, un enseignement des principes généraux du christianisme ne saurait en donner qu'une version édulcorée, écartant la doctrine, les traditions, les rites et les dogmes du catholicisme qui sont essentiels à la foi des fidèles. De plus, pour $\mathrm{M}^{\mathrm{gr}}$ de Charbonnel, la mixité religieuse soumettrait les enfants catholiques à l'influence d'un enseignement séculier qui les exposerait «à des interprétations bâtardes " de la vérité religieuse et à " la ruine de la religion et une persécution de l'Église. ${ }^{7}$ "En 1850, lors des débats entourant un nouveau projet de loi scolaire, M $^{\mathrm{gr}}$ de Charbonnel réclame que les droits des catholiques soient respectés et que ceux-ci obtiennent les mêmes concessions obtenues par les protestants au Canada-Est :

Those portions [catholiques] of the Upper Canadian population which are in communion with the See of Rome have ever been most desirous of having the education of their youth conducted upon such principles as will ensure to them the intimate blending of religious teaching with secular learning; in mixed schools this is obviously impossible. [...] the Catholic community of Upper Canada do not desire to stand up stiffly for their strict legal rights, when a harmonious understanding can be attained; they have every disposition to meet their separated Brethren upon equitable terms, and thereby avert the clashing and jarring which might ensue to their mutual great inconvenience, where the law to be rigidly followed out in all its details. ${ }^{8}$

Enfin, pour Charbonnel et ses successeurs, l'école catholique possède un caractère résolument social et communautaire. Elle fait partie intégrante de la paroisse où s'exerce le magistère de l'Église catholique par l'entremise du prêtre qui s'assure que les rites, les dévotions, les traditions et les dogmes du catholicisme soient inculqués, observés et respectés par ses ouailles. ${ }^{9}$ C'est là tout le contraire de la vision de Ryerson qui considérait que l'école devait offrir une formation morale et civique fondée sur " the general system of truths and morals taught in the Holy Scriptures " et laisser aux parents et à la communauté la responsabilité d'enseigner « the peculiarities of a sect. ${ }^{10}$ "

Les catholiques peuvent compter sur un atout majeur, soit l'appui des Canadiens français, en raison de la structure politique de la colonie qui favorise les alliances entre les représentants des deux Canadas, alliances qui prennent souvent une couleur religieuse. Ainsi, à la Chambre d'assemblée, les protestants du Canada-Ouest et du Canada-Est font front commun, tandis que les catholiques des deux côtés de la rivière des Outaouais s'offrent mutuellement leur appui. Grâce à l'alliance établie avec les députés catholiques du Canada-Est, les députés du Canada-Ouest parviennent à détenir une majorité simple et à obtenir les concessions législatives qui posent les jalons d'un système scolaire séparé. Les députés canadiens-français du Canada-Est et les députés catholiques du Canada-Ouest réussissent à faire adopter au Parlement du Canada-Uni, en 1853, en 1855 et en 1863 principalement des mesures législatives 
qui lèvent les barrières qui empêchaient les catholiques de mettre sur pied leurs propres écoles. Désormais, ceux-ci disposent du droit d'établir et d'administrer leurs écoles, de verser leurs taxes scolaires aux écoles séparées, ces dernières ayant accès aux subventions gouvernementales. ${ }^{11}$

À la suite des négociations entourant le projet confédératif, les catholiques du Canada-Ouest et les protestants du Canada-Est parviennent à faire inscrire dans la constitution une clause particulière sur les droits scolaires des minorités. L'article 93 de l'Acte de l'Amérique du Nord britannique (AANB) assure que les lois scolaires adoptées au Canada-Ouest et au Canada-Est continueront d'être en vigueur et seraient reconnues dans les nouvelles provinces. Cependant, jusqu'aux années 1960, les écoles séparées seront considérées comme un mal nécessaire par les élites politiques et éducatives ontariennes qui feront une interprétation prudente de l'article 93 lors de l'adoption des lois scolaires, laquelle traduit leur opposition de principe aux écoles séparées.

\section{Portée et limites du concordat scolaire ontarien}

Le gouvernement provincial et la majorité protestante n'ont jamais caché leur préférence pour le système scolaire public. Par la suite, les écoles séparées sont considérées par les législateurs et les élites dirigeantes comme un moindre mal pour conserver l'harmonie de la société et elles seront bon gré mal gré tolérées dans le paysage politique ontarien. Egerton Ryerson, lui-même, avait reconnu l'école séparée à contrecœur. Il ne s'est jamais caché qu'il entrevoyait le caractère temporaire de ces écoles séparées qui, tôt ou tard, " die out, not by force of legislative enactment, but under the influence of increasingly enlightened and enlarged views of Christian relations, rights and duties between different classes of community. ${ }^{12}$ " Fidèles à la pensée de Ryerson, les législateurs ontariens renforcent le statut " officiel " de l'école publique qui se concrétise par une reconnaissance politique et un accès étendu aux ressources financières, alors que le champ d'intervention des écoles séparées demeure contenu dans le cadre des lois adoptées avant la Confédération et ce, sans qu'il y ait de changements importants, avant les années 1960. Les politiciens ontariens s'engagent à ne pas remettre en question les acquis des écoles séparées, mais se montrent tout à fait opposés à accorder de nouvelles concessions financières aux catholiques. ${ }^{13}$

De prime abord, le mode de partage de la taxe scolaire avantage le système scolaire public qui peut compter sur un nombre plus important de contribuables bien nantis que le système scolaire séparé. Dans ce cas, les écoles catholiques se retrouvent avec moins d'argent par élève que les écoles publiques. Ainsi, en 1913, le Toronto Separate School Board (TSSB) dépense 18,53 \$ par élève contre 37,58 \$ pour le Toronto Board of Education (TBE). En 1924, chaque élève du TSSB reçoit 38,15 \$ contre $86,92 \$$ pour ceux du TBE (voir graphique 1 )..$^{14}$ De plus, les subventions gouvernementales sont versées aux commissions scolaires en proportion de leurs dépenses. Les commissions scolaires les moins fortunées perdent au change avec ce mode d'attribution des octrois gouvernementaux. Ainsi, à Toronto, jusqu’aux années 1960, les écoles séparées ne reçoivent pas une part des subventions gouvernementales correspondant à 
la proportion des élèves catholiques au sein de la population scolaire de la métropole. Les écoles publiques retirent la quasi-totalité des revenus des taxes scolaires prélevées sur les compagnies commerciales et les sociétés qui représentent une manne financière, surtout au tournant $\mathrm{du} \mathrm{XX}^{\mathrm{e}}$ siècle avec le développement industriel de la province et de la métropole. La loi, adoptée en 1886, permet aux directeurs de compagnie, sans que ce soit obligatoire, d'accorder une partie des revenus de la taxe scolaire aux écoles séparées correspondant à la proportion des propriétaires ou des actionnaires catholiques. Dans les faits, les écoles séparées reçoivent une fraction infime des taxes des compagnies et des sociétés (environ un pour cent). Grâce à l'accès aux taxes des sociétés et des compagnies commerciales, les écoles publiques peuvent maintenir le taux de la taxe scolaire plus bas que les écoles séparées et dépenser plus d'argent par élève. En 1921, le taux de la taxe scolaire des écoles séparées à Toronto s'élève 1,20\$ par 100 \$ d'évaluation foncière contre 0,80 \$ pour les écoles publiques. Douze ans plus tard, en 1933, le taux est respectivement pour les écoles séparées et publiques de $1,15 \$$ et de $0,70 \$$ (voir tableau 1). Malgré les sacrifices consentis par les contribuables catholiques, le revenu par élève des écoles séparées de Toronto demeure inférieur à celui des écoles publiques, et ce, jusqu’à la fin des années 1960.15

Enfin, les catholiques n'ont pas le même accès aux études secondaires. La loi sur la création des premiers high schools, adoptée en 1871, ne fait pas mention d'écoles secondaires catholiques. Les catholiques doivent s'en remettre aux écoles secondaires privées mises sur pied et administrées par l'Église catholique et les congrégations religieuses. Pour encourager l'éducation en milieu rural, le gouvernement ontarien autorise, à partir du $\mathrm{XX}^{\mathrm{e}}$ siècle, les commissions scolaires à implanter des classes de $9^{\mathrm{e}}$ et de $10^{\mathrm{e}}$ année en allouant certaines subventions. Cependant, le reste du cours secondaire $\left(11^{\mathrm{e}}, 12^{\mathrm{e}}\right.$ et $13^{\mathrm{e}}$ année) offert dans les établissements catholiques n'est pas admissible aux octrois gouvernementaux. ${ }^{16}$

\section{Mobilité sociale, éducation et droits civiques}

Entre les années 1890 et 1920, la minorité catholique de Toronto connaît un important processus de mobilité sociale qui amène ses représentants à contester le concordat scolaire qui fait des écoles séparées, selon eux, le parent pauvre du système scolaire ontarien. Au cours de cette période, les catholiques quittent les emplois d'ouvriers non qualifiés ou semi-qualifiés pour accéder à la classe moyenne. Ils désertent aussi les ghettos ouvriers pour des quartiers plus cossus habités par les protestants. Cette mobilité sociale multiplie les contacts avec les protestants et favorise les mariages mixtes ${ }^{17}$.

Le système scolaire séparé torontois ne reste pas à l'écart de ces transformations. Les élites éducatives catholiques opèrent au tournant du $\mathrm{XX}^{\mathrm{e}}$ siècle une transformation des écoles séparées pour rehausser la compétence professionnelle des enseignants selon les critères provinciaux et adopter des programmes d'études qui se rapprochent des exigences du ministère de l'Éducation. Ils répondent à la volonté des parents d'offrir à leurs enfants une éducation comparable et de même qualité que celle des écoles publiques pour leur permettre de se tailler une place enviable sur le marché de 
l'emploi et dans la société torontoise. Ces mesures adoptées par les élites catholiques visent bien sûr à contrer les inscriptions des catholiques dans les écoles publiques, mais aussi à rendre les écoles séparées admissibles aux subventions gouvernementales qui leur étaient partiellement refusées sous prétexte des programmes d'études non reconnus et du niveau de compétence jugé inférieur des enseignants ${ }^{18}$. Toutefois, les restrictions persistantes pesant sur les écoles séparées et consacrant leur infériorité financière sont perçues par la minorité catholique comme une négation du principe d'égalité des chances qui fait obstacle à ses aspirations sociales. Sous l'égide de l'archevêché de Toronto et des organisations laïques, des campagnes de «droit civique » sont menées auprès du gouvernement pour exiger l'adoption d'un mode de financement scolaire équitable.

$\mathrm{Au}$ lendemain de la Première Guerre mondiale, l'archevêque de Toronto, Neil McNeil, rappelle que les temps ont changé et lance un appel aux catholiques à l'unité pour faire reconnaître leurs droits scolaires :

They [catholiques] secured the right to Separate Schools, and this was a great religious gain; but the arrangement for the financial support of these Schools was not based on the educational needs of Ontario. It was not assumed by the Legislature that this branch of the Public School System had a citizenship value for the Province as a whole. Separate Schools could not have been secured on this basis. Anti-catholic prejudice was too strong for that. The only basis obtainable was the financial capacity of Catholics as individuals who might wish to support such schools. [...] This money, or a large public of it, would now be available for the higher education of Catholics if the public opinion admitted the fact that the Separate Schools are an efficient instrument for the training of Ontario Citizens. We have passed the test of war. No one can claim, and I do not know of any who do claim, that graduates of the Separate Schools in Ontario lacked either patriotism in enlisting or courage in battle. [...] But the time has now come for greater united efforts. The need for both the Church and the Country calls for greater efforts. ${ }^{19}$

Dans les années 1920, des groupes de pression catholiques tentent de contester devant les tribunaux la validité des lois ontariennes qui refusent d'accorder aux écoles séparées des subventions pour leurs établissements d'enseignement secondaire. Finalement, en 1928, la cause (The Tiny Township Case) se rend jusqu'au Conseil privé de Londres, l'instance judiciaire suprême de l'Empire britannique, qui n'impose pas au gouvernement provincial l'obligation de subventionner les établissements secondaires catholiques. ${ }^{20}$

Dans les années 1930, le Catholic Taxpayer's Association, fondé par l'homme d'affaires Martin Quinn, tente de faire reconnaître l'accès des écoles séparées aux taxes scolaires des compagnies commerciales et des sociétés. L’une des stratégies employées vise à mobiliser le vote catholique lors des élections pour forcer les politiciens à se positionner sur la question. Quinn trouve un terrain d'entente avec le premier ministre Hepburn qui cherche à séduire l'électorat catholique et s'engage à déposer un projet 
de loi à l'Assemblée législative. L'incapacité à parvenir à un consensus politique en raison de l'opposition d'une partie importante de l'électorat protestant a raison du projet de loi et convainc le gouvernement libéral Hepburn de reculer sur cette question. ${ }^{21}$

\section{Les écoles séparées ontariennes dans l'après-guerre Un acteur incontournable}

L'après-guerre a constitué un véritable incubateur pour les transformations sociales qui s'avéreront déterminantes pour les écoles séparées à partir des années 1960. Autrefois, l'école séparée représentait presque un mal nécessaire dans le paysage scolaire ontarien pour être graduellement acceptée par la majorité protestante. Nous pourrions presque parler d'une " révolution des mentalités " qui, toutefois, ne s'est pas accomplie sans heurts, alors que la question du financement des écoles séparées ne connaît son dénouement qu'à l'aube de l'an 2000. Cette reconnaissance des écoles séparées par la société civile ontarienne tient à plusieurs raisons.

Le processus d'intégration au sein des sociétés torontoise et ontarienne et la mobilité sociale des catholiques ont favorisé le décloisonnement des espaces ethniques et confessionnels et contribué à une meilleure compréhension mutuelle des catholiques et des protestants. Ce brassage social a permis de sortir de la logique de confrontation qui caractérisait les relations entre les deux principaux groupes confessionnels et la minorité catholique a pu désormais asseoir plus facilement la légitimité des écoles séparées, qui deviennent avec le temps un phénomène davantage toléré dans le paysage scolaire ontarien. L'immigration, au lendemain de la Seconde Guerre mondiale, constituée en grande partie de catholiques, assure l'expansion du Toronto Separate School Board qui devient une force sur laquelle le gouvernement et la société civile torontoise et ontarienne doivent compter et même s'appuyer pour intégrer les nouveaux arrivants. Enfin, les gains éducatifs des catholiques s'inscrivent dans un processus de libéralisation de la société canadienne et de reconnaissance des minorités. Les politiques fédérales de bilinguisme, de multiculturalisme et ultimement l'adoption de la Charte des droits et libertés influencent les politiques du gouvernement provincial ontarien et donnent beaucoup plus de poids aux revendications de la minorité catholique.

Dans l'après-guerre, les écoles séparées doivent relever de nouveaux défis, particulièrement à Toronto. La prospérité de la décennie suivante attire de nombreux immigrants composés en grande partie de catholiques. Entre 1939 et 1962, les effectifs des écoles séparées à Toronto passent de 12815 à 47098 élèves, alors que ceux des écoles publiques déclinent, entre 1939 et 1949 , de 100237 à 80070 élèves pour atteindre, au début des années 1960, plus de 90000 élèves. Dans les années 1960, les élèves des écoles séparées représentent plus du tiers des effectifs scolaires de Toronto, contre un peu plus de dix pour cent vingt ans plus tôt. Pourtant, en 1944, le TBE dépense $147,57 \$$ pour chacun de ses élèves contre $76,07 \$$ pour le TSSB, soit près du double de la somme. 22

Dans les années 1940, à l'instar de nombreuses sociétés occidentales, le gouvernement provincial instaure des politiques destinées à promouvoir l'égalité des chances par l'accès à l'éducation pour, entre autres, favoriser la prospérité économique grâce à 
la formation d'une main-d'œuvre qualifiée. Ce nouveau rôle que semble vouloir jouer l'État dans le champ éducatif ramène la question du financement des écoles séparées sur la place publique. Les organisations éducatives catholiques partent en campagne pour réclamer que le " principle of equality of educational opportunity " professé par le gouvernement provincial, vaille aussi pour les écoles séparées. Or, ce principe, dans l'esprit de ces organisations, ne peut se réaliser que par la reconnaissance des droits éducatifs des catholiques et l'égalité financière des écoles séparées avec les écoles publiques. Au cours des décennies qui suivront, les représentants de la minorité catholique défendront l'idée que l'école séparée est une « école publique » et doit être financée au même titre que les écoles publiques ontariennes.

Lors de la Commission royale d'enquête sur l'éducation-la Commission Hope - mise sur pied par le gouvernement conservateur Drew, en 1945, le TSSB présente un mémoire qui brosse le portrait des écoles séparées et tente de montrer leur caractère public:

In placing our children in Separate Schools we do not thereby separate ourselves from the rest of the nation, either in fact or in spirit. We follow the same course of study as do pupils in the general Public Schools; our teachers are graduates of the Ontario Normal School and the College of Education and have precisely the same qualifications as other teachers in the Province. Quite a number of them hold university degrees and many hold special certificates in various subjects, obtained through attendance at summer courses provided by the Ontario Department of Education. Thus we are an organic part of the Public School System of the Province of Ontario, both in practice and in full accordance with Ontario educational laws. We are training our children to fulfill their duties to the state and to live in co-operation and concord with their fellow citizens of other religious faiths. ${ }^{23}$

La question du financement des écoles séparées devient une véritable boîte de pandore pour le gouvernement, alors que les catholiques composent au lendemain de la Seconde Guerre mondiale un cinquième des populations ontarienne et torontoise. Le rapport majoritaire de la Commission Hope, déposé en 1950, propose un financement complet et équitable des écoles séparées jusqu'à la $6^{\mathrm{e}}$ année. Toutefois, le refus d'étendre le financement aux high schools catholiques force les membres catholiques de la Commission Hope à publier un rapport minoritaire et dissident. Le premier ministre conservateur Frost, conscient des risques politiques d'adopter la ligne dure, choisit d'augmenter les octrois gouvernementaux aux écoles séparées afin d'éviter ainsi de s'aliéner une partie de l'électorat peu favorable à une réforme du financement scolaire à l'intention des catholiques. ${ }^{24}$

C'est cette politique qui prévaudra au sein des divers gouvernements. À la demande des associations éducatives catholiques, le gouvernement Robarts adopte au cours des années 1960 divers programmes comme le " equalized assessment ", " growth-needs factor» et "Ontario Foundation Tax Plan ». Ces mesures visent à injecter des sommes d'argent pour réduire l'écart des revenus et la disparité des services 
éducatifs entre les commissions scolaires plus riches et plus pauvres. ${ }^{25}$ L'Ontario Foundation Tax Plan notamment dispose de l'avantage de promouvoir l'accessibilité à l'éducation sans favoriser explicitement une catégorie de la population bien que les commissions scolaires séparées en sont les principales bénéficiaires en raison de la faiblesse de leurs revenus. Entre 1960 et 1967, la somme d'argent dépensée par élève dans les écoles séparées de Toronto passe de 151,27 à 434,08 \$, alors que celui des écoles publiques croît de 340,59 \$ à 466,12 \$ avant d'atteindre la parité dans les années $1970 .^{26}$

Cependant, la question de subventionner les écoles séparées par d'autres sources que les taxes scolaires payées par les contribuables catholiques ne fait pas l'unanimité dans les années 1960. Lors d'un débat l'opposant au commissaire du TSSB, Edward J. Brisbois, dans les pages du Toronto Star, F. L. Bartlett, professeur à l'Université Queen's et président du Ontario School Trustees' Council résume le point de vue qui devait sans doute être partagé par de nombreux protestants à l'époque :

It is essential to remember, regarding sources of revenue for the support of separate schools that they are denominational schools and what is being considered is the support of schools operated for the benefit of one religious denomination. Some Protestants feel they are caught in a conflict of two basic principles: First, that equality of educational opportunity must be extended to all children; and second, that no person of one religious conviction should be taxed directly or indirectly for the support of denominational schools for a person of a different religious conviction. In short, while public school supporters are mainly willing to pay for the secular education of all children on an equal basis, they are not willing to pay denominational instruction of children of any specific religious group. ${ }^{27}$

D'autre part, la question de l'accès des high schools catholiques aux subventions gouvernementales reste entière et leur coût de fonctionnement demeure en grande partie assumé par les commissions scolaires séparées grevant leur budget. Les nombreuses campagnes publiques des organisations catholiques en faveur d'un financement intégral des high schools catholiques se heurtent à l'opposition du gouvernement et d'une partie de l'opinion publique. À la surprise générale, le premier ministre Bill Davis, reconnu pour être intraitable sur cette question, adopte une loi, en 1984, juste avant de quitter la vie politique, qui étend le financement gouvernemental aux high schools catholiques. $^{28}$

En 1997, le gouvernement conservateur Harris adopte la loi 160 qui prévoit la réduction du nombre des commissions scolaires et des commissaires, fixe un palier pour les salaires et, innovation marquante, abolit la taxe scolaire et accorde à l'État la responsabilité de financer les écoles. Cette mesure consacrait l'égalité financière entre les écoles séparées et publiques tant réclamée par les catholiques, mais la "prise en charge " du financement par le gouvernement a inspiré des inquiétudes pour certains d'entre eux qui craignaient que l'État resserre son contrôle et en profite pour remettre en question le statut confessionnel des écoles séparées. 


\section{Le système scolaire protestant au Québec Un État dans un État?}

Au cours des années 1840 et 1850, les élites réformistes du Canada-Est posent les premiers jalons d'un système scolaire public et non confessionnel destiné à refléter les valeurs libérales d'accessibilité à l'éducation, de démocratisation politique et de progrès économique. Des penseurs libéraux comme le surintendant de l'éducation, Jean-Baptiste Meilleur et Pierre-Joseph-Olivier Chauveau, futur premier ministre de la province de Québec, considèrent que l'instruction publique doit viser à former des citoyens exemplaires, conscients de leurs devoirs civiques, qui participeront à la vie politique d'un gouvernement représentatif. De plus, l'instruction publique est vue comme un préalable essentiel à la prospérité économique en préparant des travailleurs qualifiés au marché de l'emploi. ${ }^{29}$

Cette conception libérale sera battue en brèche par l'Église catholique, qui ne cache pas ses prétentions sur l'éducation. Selon la pensée ultramontaine, le système scolaire a pour fonction de " christianiser " et de " moraliser " les enfants afin d'en faire des chrétiens respectueux de l'autorité et de l'ordre établi, de définir les normes sociales d'après ses préceptes idéologiques et de façonner la culture religieuse des masses canadiennes-françaises. La hiérarchie catholique voit l'école " commune ", promue par les libéraux, comme un produit dérivé du protestantisme. Elle privilégie une confessionnalité catholique et dénonce avec virulence " tout système d'éducation élémentaire, qui réunit sous un même toit et sous la férule du même maître les enfants de différentes croyances » et qui " doit nécessairement amener le mépris pour tous les dogmes et conduire les élèves à l'indifférence absolue. ${ }^{30}$ " De leur côté, les protestants du Canada-Est tendent à se méfier d'une école qui serait inévitablement dominée par les catholiques majoritaires au Bas-Canada. Ils invoquent le principe de la confessionnalité pour s'assurer du contrôle de leurs propres écoles. ${ }^{31}$

Pour vaincre les résistances à la fois des catholiques et des protestants à son projet éducatif, le gouvernement adopte une loi, en 1841, qui établit des écoles "communes " - sans caractère confessionnel — tout en insérant une clause de dissidence qui permet à un groupe religieux minoritaire de mettre sur pied à certaines conditions ses propres écoles. Peu à peu, le principe de la confessionnalité se glisse à travers cette embrasure et se généralise à l'ensemble du système scolaire. À l'extérieur de Montréal et de Québec, où les commissions scolaires sont confessionnelles, les écoles de la majorité deviendront catholiques et celles de la minorité se constitueront en écoles protestantes, sauf dans les régions où les protestants sont majoritaires comme en Outaouais et dans les Cantons de l'Est. ${ }^{32}$

Au cours de la décennie suivante, les débats politiques entourant le projet confédératif tiennent le haut du pavé. En octobre 1864, lors de la Conférence de Québec, les délégués s'entendent pour que dans le nouveau régime politique l'éducation soit une compétence provinciale. Cette annonce a l'effet d'une bombe parmi les représentants de la minorité anglophone, qui craignent d'être livrés à la merci de la majorité francophone. ${ }^{33}$ Alexander Tilloch Galt, ministre des Finances au sein du gouvernement du Canada-Uni et ardent partisan de la Confédération, met sa carrière en jeu pour 
s'assurer que les acquis scolaires des protestants du Canada-Est soient préservés dans le nouveau régime politique. ${ }^{34}$ Le 23 novembre 1864, dans un discours à Sherbrooke, Galt prévient ses coreligionnaires du danger qui les guette :

It must be clear that a measure would not be favorably entertained by the minority of Lower Canada, which would place the education of their children in the hands of a majority of a different faith. It was clear that in confiding the general subject of education to the local legislature, it was absolutely necessary it should be accompanied with such restrictions as would prevent injustice in any respect being done to the minority. ${ }^{35}$

Déjà au mois de septembre 1864, des représentants de l'éducation avaient fondé le Lower Canadian Protestant Education Society qui avait formulé les revendications éducatives suivantes: « a separate department of education for Protestant children, a more convenient arranging of Protestants school districts, the assurance that in no case would Protestant be taxed for Catholic Schools even where there was no Protestant School, and special government grants for Protestant universities, normal schools, colleges, academies in high schools ${ }^{36}$ ". Ces demandes visent essentiellement à ériger un système scolaire protestant indépendant vis-à-vis du futur État provincial dirigé en majorité par les catholiques et les francophones, pour se soustraire notamment à l'influence ultramontaine de l'Église catholique dans le champ éducatif. Le retrait progressif de l'État du champ de l'éducation et de son corollaire incontournable, la confessionnalisation du système scolaire, rendra possible le programme projeté par les élites éducatives protestantes. ${ }^{37}$

En décembre 1866, lors de la Conférence de Londres, grâce aux efforts infatigables de Galt, les protestants du Canada-Est obtiennent que leurs "droits et privilèges " scolaires consentis sous le régime de l'Union soient maintenus - ainsi que ceux des catholiques du Canada-Ouest. Coup de maître, Galt parvient à faire accepter une clause capitale pour les droits des minorités qui accorde le pouvoir au gouverneur général d'invalider une loi provinciale qui ne respecterait pas les droits scolaires consentis aux minorités catholique et protestante des provinces de l'Ontario et du Québec permettant ainsi au parlement fédéral de décréter sa propre loi. ${ }^{38}$

Au lendemain de la Confédération, le gouvernement québécois se désengage progressivement du champ de l'éducation pour en confier la gestion à l'Église catholique et aux élites éducatives protestantes. Sitôt nommé premier ministre de la province par l'Assemblée législative lors de la première session parlementaire, Pierre-JosephOlivier Chauveau, doit composer avec les revendications de l'Église catholique, qui souhaite accroître son ascendant sur le système d'éducation, et des élites éducatives protestantes qui réclament la reconnaissance de garanties scolaires pour la minorité. De plus, il est lié par la promesse de George-Étienne Cartier, chef des conservateurs du Canada-Est qui s'est engagé à satisfaire les demandes éducatives des protestants advenant l'adoption de la Confédération.

Ainsi, en 1869, le gouvernement Chauveau adopte une loi censée satisfaire toutes les parties. Le système scolaire est scindé en deux blocs distincts fondés sur 
l'appartenance religieuse par la création des comités confessionnels au sein du Conseil de l'Instruction publique et qui sont pratiquement souverains dans leurs champs de compétences respectifs. Ainsi, les politiques scolaires relèvent peu à peu de la responsabilité des comités confessionnels, affaiblissant ainsi les pouvoirs du ministère de l'Instruction publique créé l'année précédente. Cependant, l'une des innovations les plus importantes de la Loi demeure la création d'une taxe scolaire à Montréal. ${ }^{39}$ L'abolition du ministère de l'Instruction publique en 1875 sonne le glas des prétentions de l'État et laisse en matière scolaire les coudées franches à l'Église catholique et aux élites éducatives protestantes. Cette loi remplace le ministre de l'Instruction publique par un surintendant qui dispose d'un pouvoir désormais amenuisé vis-à-vis des comités confessionnels du Conseil de l'Instruction publique qui ont l'autorité presque exclusive pour administrer les écoles de leurs coreligionnaires respectifs. ${ }^{40}$

Force est de constater que le rôle de l'État en ce qui touche l'éducation au Québec a suivi une évolution très différente de celle de l' "Educational State " en Ontario. Désormais, le gouvernement québécois distribue les subventions, entérine et coordonne les décisions prises par chacun des deux comités confessionnels et agit parfois à titre d'arbitre en cas de conflits entre catholiques et protestants. Il se garde bien d'intervenir dans le champ scolaire dévolu à la majorité et confié à l'Église catholique ou dans celui de la minorité protestante et ne fait pratiquement pas obstacle à leurs décisions.

\section{Le concordat scolaire de 1869 , un compromis honorable?}

À première vue, les protestants semblent avoir obtenu gain de cause par la reconnaissance du gouvernement provincial de la plupart de leurs revendications éducatives. Il faut plutôt voir la loi de 1869 comme un compromis établi par le gouvernement Chauveau pour satisfaire les ultramontains et les protestants et comprenant des gains et de concessions pour les deux parties. D'emblée, Chauveau reconnaît l'une des revendications principales des protestants, celui de diviser les revenus de la taxe scolaire à Montréal d'après la religion des propriétaires. Les protestants trouvaient odieux que leur argent puisse contribuer à financer des écoles catholiques qui enseignent une doctrine contraire à leurs convictions religieuses. Or, le niveau de richesse des protestants, plus élevé que celui des catholiques, permet au Protestant Board School Commissioners of the City of Montreal (PBSCCM) de dépenser plus d'argent par élève que la Commission des écoles catholiques de Montréal (CECM). En 1899, le PBSCCM peut consacrer 19,32 \$ à chacun de ses élèves et la CECM à peine $9,80 \$$ (voir graphique 1). ${ }^{41}$ Bien que les protestants ne constituent que le quart de la population de Montréal, le PBSCCM retire autant d'argent de la taxe scolaire que la CECM. ${ }^{42}$ Pendant l'entre-deux-guerres, bien que la proportion de l'écart se rétrécisse, l'inégalité persiste. Entre 1922 et 1942, le montant d'argent dépensé pour chaque élève protestant au PBSCCM passe de 60,42 à 115,26 \$ contre respectivement 48,59 \$ à 85,86 $\$$ pour les élèves catholiques de la CECM. ${ }^{43}$

Le gouvernement Chauveau divise les revenus des taxes scolaires prélevées sur les sociétés et les compagnies commerciales proportionnellement à la population 
catholique et protestante. Dans ce cas-ci, les protestants ont essuyé un refus net de la part du gouvernement, alors qu'ils exigeaient que la taxe scolaire dite " des neutres » soit divisée selon la religion des propriétaires et des actionnaires. Dans les années 1880 et 1890, frappées de plein fouet par la crise économique, les commissions scolaires montréalaises sont à la recherche de nouvelles sources de financement. Les commissaires protestants réclament à plusieurs reprises que les taxes scolaires dites " des neutres » soient divisées entre le PBSCCM et la CECM d'après la religion des actionnaires et des propriétaires et que les taxes scolaires payées par tous les propriétaires non catholiques soient versées obligatoirement au fonds des écoles protestantes. Les menaces du comité catholique du Conseil de l'instruction publique et de la CECM d'instaurer un mode de répartition de l'ensemble des revenus scolaires au prorata des populations catholique et protestante font reculer le PBSCCM sur cette question. Cet épisode montre bien que les élites politiques, religieuses et éducatives canadiennes-françaises jugeaient que le pacte scolaire de 1869 possédait un caractère permanent et définitif. ${ }^{44}$

Enfin, le mode de distribution pour les subventions gouvernementales est adopté cette fois-ci à l'avantage des établissements catholiques d'enseignement supérieur. Les protestants réclamaient que les octrois gouvernementaux consacrés aux établissements d'enseignement supérieur soient répartis d'après «[the] amount contributed by the people, the number of pupils in attendance, the duration of the schools, and the character of the instruction given. ${ }^{45}$ ". Rappelons qu'à l'époque, l'enseignement supérieur comprenait les écoles modèles, les académies, les collèges classiques et commerciaux, les high schools et les universités. Chauveau veut sans doute rallier l'opinion publique canadienne-française qui pouvait considérer comme excessives les concessions accordées aux protestants. Désormais, les subventions du Fonds d'éducation supérieure seront distribuées au prorata des populations catholique et protestante, plutôt que d'après le nombre d'établissements ou du nombre d'étudiants comme c'était le cas auparavant. Mieux organisés institutionnellement et plus scolarisés que les catholiques, les protestants étaient avantagés par l'ancienne formule. Ainsi, en 1868, à Montréal, les établissements scolaires protestants recueillaient 6276,22 \$ du Fonds d'éducation supérieure et ceux des catholiques, 3197,38 \$. Pourtant, les protestants ne composent que le tiers de la population de Montréal. L'année suivante, le nouveau partage du Fonds d'éducation supérieure accorde aux établissements catholiques $6489,45 \$$, tandis que ceux des protestants recueillent $2405,55 \${ }^{46}$

Ce concordat scolaire permet de parvenir à un modus vivendi viable, durable et acceptable entre les deux principaux groupes confessionnels. Les élites politiques et religieuses canadiennes-françaises saluent les subventions accordées aux collèges classiques, tandis que les protestants disposent désormais de l'autonomie financière, pédagogique et administrative nécessaire pour établir leur propre système scolaire public qui évoluera indépendamment de celui de la majorité. En somme, les élites canadiennesfrançaises et anglo-protestantes vivent tant bien que mal une situation qu'elles jugent plus ou moins satisfaisante, mais qui permet de tempérer un conflit qui menaçait la bonne entente entre les deux principaux groupes confessionnels. C'est le journal $\mathrm{La}$ Minerve qui résume probablement le mieux la volonté des législateurs de parvenir à un 
compromis scolaire entre les deux principales collectivités religieuses et linguistiques :

Le bill de l'éducation est une mesure de concessions et l'on doit se réjouir de son succès, du moment que ceux pour qui il a été fait, dans un but de paix et de conciliation, s'en montrent satisfaits et contents. Un gouvernement qui peut se vanter d'avoir assuré la concorde parmi les dénominations religieuses se vante d'une grande chose. Ces concessions ont été franches, sincères et elles n'ont pas dépassé les bornes de la raison. On demandait beaucoup de nous; nous l'avons accordé en demandant un peu en retour comme réciprocité de la libéralité. On voulait une loi juste pour tous, et ceux qui demandaient la disparition de certains désavantages contre la minorité ne pouvaient que se montrer prêts à renoncer au maintien de certains désavantages contre la minorité. On voulut donc des deux côtés et d'un commun accord que la réorganisation du Département de l'Instruction publique et la répartition des taxes scolaires rencontrent mieux les idées des protestants; mais, en même temps, on convint que la révision des octrois pour l'éducation supérieure fit disparaître la disproportion entre les protestants et les catholiques. ${ }^{47}$

Il reste qu'à travers ce compromis les élites canadiennes-françaises s'en remettent en partie aux institutions privées du clergé catholique, jugées plus prestigieuses et moins coûteuses que celles de la CECM. De fait, jusqu'à la Seconde Guerre mondiale, les contribuables francophones se montreront hostiles à une hausse du taux de la taxe scolaire, ainsi qu'à toute initiative de l'État visant à allouer plus de ressources financières à la CECM, considérant que les congrégations religieuses peuvent offrir les mêmes services et à moindre coût sans que leur fardeau fiscal soit augmenté. Bien servies par les institutions privées, les élites canadiennes-françaises ne se sont guère préoccupées d'assurer aux écoles publiques un financement adéquat et encore moins d'instituer une filière secondaire dans les commissions scolaires avant les années 1920.

C'est tout le contraire des élites éducatives protestantes qui prônent l'élaboration d'un réseau public d'écoles qui assurerait la scolarisation de l'ensemble de la population protestante pour maintenir leur influence et éviter d'être délogé des sphères sociales, économiques et politiques par les Canadiens français qui les surpassent en nombre. Ainsi, tout au long du dernier tiers du XIX ${ }^{\mathrm{e}}$ siècle, le PBSCCM ne cesse de presser le gouvernement provincial de modifier la législation scolaire à son avantage pour renforcer son autonomie financière et administrative vis-à-vis des pouvoirs publics dominés par les francophones. De plus, scolarisée dans sa quasi-totalité par le secteur public, la population protestante consent aisément à des sacrifices lorsque le PBSCCM demande une hausse du taux de la taxe scolaire. Et c'est pourquoi tout au long de la période étudiée et jusqu'aux années 1960, le taux de la taxe scolaire des contribuables protestants demeure presque toujours plus élevé que celui des contribuables catholiques. Ainsi, dans les années 1920, le taux de la taxe scolaire des protestants s'élève à $1,00 \$$ pour $100 \$$ d'évaluation foncière contre $0,70 \$$ pour celui des catholiques. Dans les années 1950, il atteint pratiquement le double : 1,50 \$ pour les protestants contre $0,80 \$$ pour les catholiques (voir tableau 2). 
Il reste, bien que cela puisse paraître paradoxal, que pour de nombreux dirigeants scolaires protestants, il n'y a pas lieu de pavoiser. Malgré les gains obtenus, les sommes d'argent accordées aux protestants sont nettement insuffisantes pour assurer leur progrès éducatif. Ils ne peuvent compter que sur eux-mêmes, insiste John W. Dawson, principal de l'Université McGill :

The Protestants were a small, and, as some said, a decaying people in this province, but they were bound to uphold their system of education by all means in their power. A larger sum was necessary for the support of elementary schools, and it had been suggested that an increased grant be asked for. That would be better in some respects and worse in others, for out of the extra sum raised only one-sixth or one-tenth would come to us. We paid more than the share we got, and if more was necessary, we would do better to raise it ourselves. ${ }^{48}$

\section{L'école secondaire publique, porte d'entrée à la contestation}

La question de l'enseignement secondaire public jouera un rôle fondamental dans la critique du mode de financement scolaire qui mènera à sa démocratisation dans les années 1960 et 1970 . À cet égard, le PBSCCM fait figure de pionnier. Grâce à un partage avantageux de la taxe scolaire, le PBSCCM investit le champ de l'enseignement secondaire par l'acquisition d'une institution privée reconnue, le High School of Montreal, en 1870 et la fondation du High School for Girls, en 1875. Du côté catholique, l'enseignement secondaire relève essentiellement des établissements privés - les collèges commerciaux, les pensionnats pour jeunes filles et les collèges classiques - détenus par le clergé et par les religieuses.

Au tournant du $\mathrm{XX}^{\mathrm{e}}$ siècle, les libéraux réclament des réformes éducatives pour permettre à la société de relever les défis d'une économie industrielle et d'assurer l'émancipation économique des Canadiens français. Ils proposent, entre autres, la création d'écoles techniques, l'inauguration d'un enseignement professionnel et l'instauration d'une filière secondaire publique. Des réformes auxquelles s'opposent certains membres du clergé et de la petite bourgeoisie qui craignent que l'accès des classes populaires aux études supérieures mine l'ordre social et menace le monopole des collèges classiques sur l'enseignement secondaire. Après deux décennies de débats, le Comité catholique de l'Instruction publique adopte, en 1929, un programme d'études qui inaugure le primaire complémentaire et le primaire supérieur pour préparer essentiellement ses diplômés aux métiers qualifiés et aux carrières de commerce, des sciences ou de génie. Le baccalauréat ès arts, qui comprend l'enseignement du latin, décerné par les collèges classiques, donne accès aux facultés les plus prestigieuses (droit, médecine et théologie).

Cette préférence des élites canadiennes-françaises pour les établissements privés se manifeste aussi par la distribution des octrois gouvernementaux entre les écoles primaires supérieures et les collèges catholiques. Entre les années 1920 et 1960, les collèges catholiques reçoivent la plus grande partie des sommes du Fonds de l'éducation 
supérieure même si les effectifs du secondaire public s'avèrent plus élevés à partir de la Seconde Guerre mondiale, et ce, jusqu'aux années 1960 (voir graphique 3). ${ }^{49}$ Ainsi, en 1945, 13404 et 31396 étudiants fréquentent respectivement les collèges privés catholiques et les écoles primaires supérieures $\left(9^{\mathrm{e}}, 10^{\mathrm{e}}, 11^{\mathrm{e}}\right.$ année). Cependant, les collèges privés catholiques se voient décerner $300000 \$$ en subventions du Fonds de l'éducation supérieure contre $75000 \$$ pour les écoles primaires supérieures. ${ }^{50}$ Plus d'une décennie plus tard, en 1961, les institutions privées accueillent 32935 élèves contre 217721 élèves pour les écoles secondaires publiques ( $8^{\mathrm{e}}, 9^{\mathrm{e}}, 10^{\mathrm{e}}, 11^{\mathrm{e}}$ et $12^{\mathrm{e}}$ année). ${ }^{51}$ Toutefois, ces dernières reçoivent du gouvernement un montant de $550800 \$$, tandis que les collèges obtiennent la somme de $4123500 \$ .^{52}$ Le parti pris du gouvernement du Québec traduit le prestige et la primauté accordés à l'enseignement classique par les élites politiques et sociales canadiennes-françaises dans l'enseignement secondaire et sa volonté politique d'encourager l'expansion des établissements privés qui constitue une formule beaucoup plus économique pour les coffres de l'État. ${ }^{53}$

Malgré l'essor du primaire supérieur à Montréal pendant l'entre-deux-guerres - les effectifs scolaires passent entre 1928 et 1945 de 815 à 13775 élèves-les milieux éducatifs francophones accusent un retard sur les secteurs anglo-catholiques et protestants. En effet, l'absence du latin dans le programme d'études du primaire supérieur limite l'accès à ses diplômés aux facultés les plus prestigieuses, contrairement aux étudiants des high schools protestants. De plus, à partir de 1930, un programme d'études, inspiré des high schools protestants est adopté dans les écoles de langue anglaise de la CECM. ${ }^{54}$ Cette inégalité d'accès aux études supérieures influence de manière importante les taux de scolarisation des trois principaux groupes linguistiques et confessionnels. En 1953, environ $12 \%$ des élèves du secteur français de la CECM sont inscrits au cours secondaire contre $19 \%$ des anglo-catholiques qui fréquentent les high schools. Les élèves des high schools représentent $27 \%$ des inscriptions totales du PBSCCM. ${ }^{55}$

Cette question de l'accessibilité des étudiants aux études supérieures, conjuguée à celle d'un sous-financement chronique de cette filière d'enseignement, ne tardera pas à se politiser. Au lendemain de la Seconde Guerre mondiale, à la faveur de l'émergence d'un nouveau nationalisme, les Canadiens français du Québec se perçoivent graduellement comme une société majoritaire et délaissent les anciennes formules de compromis avec la minorité anglophone et prônent l'intervention de l'État pour se porter à la défense de leurs intérêts. Les études et les rapports des commissions d'enquête sur l'éducation témoignent de la sous-scolarisation et de l'infériorité socioéconomique des Canadiens français qui catalysent les revendications des néo-nationalistes. Les divers milieux sociaux, éducatifs et intellectuels font de la réforme éducative un enjeu vital pour la nation canadienne-française et pressent l'État d'intervenir au profit de la majorité. ${ }^{56}$

C'est dans ce contexte social et politique que les commissions scolaires montréalaises, ainsi que d'autres acteurs sociaux associés aux milieux scolaires et d'affaires, participent activement aux différentes commissions d'enquête concernant l'éducation, tenues dans l'après-guerre, pour défendre une vision plus démocratique de l'éducation. Ainsi, à la Commission royale d'enquête sur les problèmes constitutionnels du 
Québec - plus connue sous le nom de la Commission Tremblay - la CECM valorise une nouvelle conception de l'école publique " au sens où elle sera soutenue, sinon uniquement au moins en très grande partie, par le trésor public » et "Qu'une commission scolaire ne devrait pas non plus être organisée en rapport avec la fortune ou le manque de fortune des parents de la foi religieuse que cette commission scolaire représente. ${ }^{57}$ " Le rapport de la Commission royale d'enquête recommande que le gouvernement provincial accorde davantage de subventions aux commissions scolaires, mais sans remettre en question le mode de partage de la taxe scolaire. ${ }^{58}$ Cependant, comme on peut le voir au graphique 2, les espoirs suscités pour les promoteurs de l'enseignement secondaire public par le rapport favorable de la Commission Tremblay ne se concrétiseront pas. L'augmentation des subventions gouvernementales pour les établissements secondaires profite surtout aux collèges privés.

Le système scolaire protestant ne demeure pas à l'abri des défis financiers que pose l'enseignement secondaire public. Entre 1922 et 1951, les effectifs des classes secondaires quadruplent passant de 2738 à 9736 élèves. La proportion de ces derniers s'accroît au cours de cette période de neuf à vingt-trois pour cent de la population étudiante totale du PBSCCM ${ }^{59}$. Or, l'enseignement secondaire, autrement plus coûteux que celui du niveau élémentaire, exerce une pression financière sur le PBSCCM. Nous avons vu que traditionnellement le système scolaire protestant s'était développé en marge de l'État provincial grâce à une autonomie institutionnelle soutenue par un partage avantageux de la taxe scolaire, particulièrement à Montréal. Toutefois, à partir des années 1920 et 1930, les revenus de la taxe scolaire ne comblent plus les besoins du PBSCCM vis-à-vis de l'augmentation des inscriptions de ses high schools.

En 1937, le comité protestant du Conseil de l'Instruction publique met sur pied, une commission d'enquête, présidée par un éducateur écossais, W. A. F. Hepburn, qui est chargé d'enquêter sur le système scolaire protestant au Québec. Au cours de cette commission d'enquête, de nombreux groupes comme le Montreal Board of Trade et le Provincial Association Protestant Teacher (PAPT) réclament un plus grand engagement financier de l'État pour soutenir l'enseignement secondaire et un meilleur mode de financement qui ferait abstraction des distinctions confessionnelles traditionnelles. ${ }^{60}$ Le rapport Hepburn respecte les vues d'une partie grandissante de la population anglophone en préconisant une intervention financière accrue de la part de l'État et une répartition plus équitable des revenus consacrés à l'éducation entre les différentes commissions scolaires. ${ }^{61}$ Bien que le gouvernement Duplessis accorde une aide financière supplémentaire de l'État à l'éducation dans l'après-guerre, une certaine partie des élites traditionnelles protestantes dénonce ces politiques qui s'accompagnent souvent d'un droit de regard sur les dépenses des commissions scolaires. ${ }^{62}$ Pourtant, l'unanimité d'antan au sein de la minorité protestante ne règne plus. Les journaux The Montreal Gazette et The Montreal Daily Star, ainsi que les milieux d'affaires accueillent avec bienvenue les subventions gouvernementales même au prix d'une certaine autonomie institutionnelle. ${ }^{63}$ Se dessine alors un dilemme sociopolitique qui ne tardera pas à refaire surface dans les années 1960 et 1970.

À peine une décennie plus tard, à la suite de la chute du gouvernement de Duplessis et de ses successeurs, les forces réformistes trouvent une oreille attentive 
auprès du nouveau gouvernement Lesage qui, dès son entrée au pouvoir, institue une commission royale d'enquête sur l'éducation restée célèbre sous le nom de la Commission Parent. Dans les années 1960, dans un contexte de bouillonnement idéologique, social et politique, l'État intervient dans le champ éducatif en rompant avec le rôle qui avait été le sien, soit un arbitre et un gestionnaire des rapports ethniques, pour devenir un agent de justice sociale qui vise à rééquilibrer les avancées et les retards scolaires des diverses composantes de la société québécoise. ${ }^{64}$ Les rapports de la Commission Parent témoignent abondamment de la nouvelle vocation de l'État dans le champ éducatif :

[...] l'éducation ne sert pas seulement à celui qui en a bénéficié, mais aussi à la société, au progrès de laquelle il contribuera de façon plus efficace et plus active. Bref, les bénéfices de l'éducation dépassent l'individu et la localité; le progrès de l'enseignement sert principalement les intérêts généraux de la société, de sorte qu'on peut et qu'on doit désormais considérer l'éducation comme une entreprise nationale. Cela signifie que l'éducation de chaque enfant, et aussi de chaque adulte n'est plus principalement sous la responsabilité d'une collectivité locale ou de groupes particuliers; c'est la société tout entière qui est responsable de l'éducation la plus complète possible de chaque étudiant, sans égard à la région qu'il habite ou à son milieu d'origine. ${ }^{65}$

Cette nouvelle conception éducative n'est pas seulement l'apanage de la population francophone. Diverses voix, notamment les milieux d'affaires, la presse et les associations de professeurs, s'élèvent au sein de la minorité anglophone pour réclamer une plus grande contribution financière de l'État pour assurer l'avenir de son système d'éducation, alors qu'il n'est plus possible de compter uniquement sur les revenus de la taxe scolaire. Si le PBSCCM demeure jusqu'au-boutiste et s'oppose tout au long des années 1960 et 1970 à une modification de la répartition de la taxe scolaire, la presse anglophone, plus pragmatique, adhère plutôt aux principes d'une réforme du mode de financement à la condition que les sommes décernées par l'État soient augmentées pour l'ensemble des commissions scolaires. Ainsi, The Montreal Star, porteparole de la communauté anglophone, se ralliera dans le milieu des années 1960 à un mode de financement scolaire fondé sur la péréquation : "The solution in the best interest of Montreal's school children is equal per-pupil taxation revenues. This would be keeping with the principle of equal educational opportunity for all, as advocated by the Department of education and Parent Commission. ${ }^{66}$ "

Dans les années 1960, incarnant cette nouvelle vision éducative, le gouvernement provincial opère une réforme du mode de financement qui vise à donner le coup d'envoi au secteur secondaire francophone en retard sur celui du PBSCCM. Ce nouveau partage des revenus s'avère nécessaire face à la progression fulgurante des effectifs de la CECM. Entre 1963 et 1970, sa population scolaire atteint un sommet passant de 189972 à 228271 élèves, puis diminue, en 1973, à 208679 élèves, en raison du déclin de la clientèle du niveau élémentaire, alors que le secteur secondaire est en progression constante passant de 45996 à 93549 élèves. Les effectifs aux niveaux 
élémentaire et secondaire du PBSCCM se stabilisent durant toute cette période respectivement autour de 40000 et de 20000 étudiants. ${ }^{67}$

Le gouvernement provincial consacre une grande partie des ressources financières allouées à l'éducation pour mettre fin à aux disparités des revenus entre commissions scolaires francophones et anglophones. Pour ce faire, il augmente ses subventions à la CECM et réduit la proportion de la part perçue par le PBSCCM des taxes des compagnies commerciales et des sociétés au profit de la CECM. De plus, le taux d'imposition de la taxe scolaire des compagnies commerciales et des sociétés, des écoles catholiques et des écoles protestantes est sans cesse augmenté. En 1960, la CECM dépense 277,28 \$ par élève contre 382,84 \$ pour le PBSCCM. Une décennie plus tard, en 1973, la CECM rattrape le PBSCCM en déboursant 1009,53 \$ par élève contre 1006,78 \$ pour le PBSCCM. ${ }^{68}$ En 1973, le Conseil scolaire de l'île de Montréal est créé : sa fonction est de fixer la taxe scolaire à un taux uniforme, de prélever les sommes d'argent et de les répartir entre les commissions scolaires selon le nombre d'élèves et leurs besoins. ${ }^{69}$ Ce système de péréquation s'étendra peu à peu au reste de la province. Cent ans d'inégalité financière entre les deux commissions scolaires montréalaises prenaient fin. ${ }^{70}$

Au terme de ce tableau esquissé ici, nos recherches font ressortir le caractère politique de la scène scolaire qui constitue un espace de discussion, de négociation et même d'affrontement où se reflètent les rapports de force entre les différents groupes religieux et culturels qui tentent de faire valoir leurs conceptions éducatives et d'avoir accès aux ressources financières consacrées à l'éducation. Cependant, ces rapports de force ne sont pas statiques et ils évoluent au gré du changement des mentalités et des conjonctures sociopolitiques.

Après avoir accordé des privilèges aux écoles publiques qui sont refusés à la minorité catholique par crainte de miner les fondements de la société ontarienne, l'État étend ces avantages aux écoles séparées à partir des années 1960. Ces mesures s’inscrivent dans la volonté de l'État d'intégrer les groupes minoritaires marginalisés à la société ontarienne et aussi pour que le système scolaire reflète davantage dans son ensemble la diversité du tissu social.

Le Québec suit le parcours inverse. L'État, dès la deuxième moitié du XIXe siècle, se campe dans une position de non-ingérence dans les rapports ethnoreligieux en accordant l'égalité de principe et une complète autonomie aux systèmes scolaires catholique et protestant. Dans les années 1960, le compromis ne tient plus aux yeux de la majorité francophone qui en appelle à l'État pour restaurer un équilibre perdu, selon elle, depuis longtemps à l'avantage de la minorité.

Cette double évolution, à la fois similaire et opposée entre Toronto et Montréal, que nous avons tenté de retracer bien schématiquement et dont le potentiel comparatif mériterait d'être exploré davantage, nous rappelle la tâche difficile pour le gouvernement et les milieux scolaires, et ce, encore aujourd'hui, de concilier la cohésion de la société, les aspirations identitaires des divers groupes religieux ou culturels et les principes de la justice sociale. 


\section{Annexes}

\section{Graphique 1}

Revenu disponible en dollars par élève au Toronto Board of Education (TBE) et au Toronto Separate School Board (TSSB) (1882-1960) Années choisies

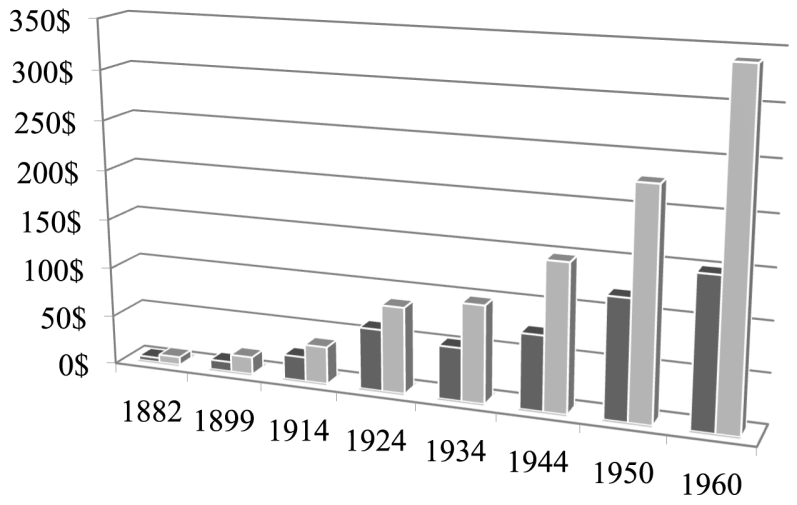

Sources: Minute Book. Toronto Catholic District School Board Archives. (TCDSBA). Minute Book. Toronto District School Board Archives. (TDSBA). 


\section{Graphique 2}

Revenu disponible en dollars par élève à la Commission des écoles catholiques de Montréal (CECM) et au Protestant Board School Commissioners of the City of Montreal (PBSCCM) (1882-1960)

Années choisies

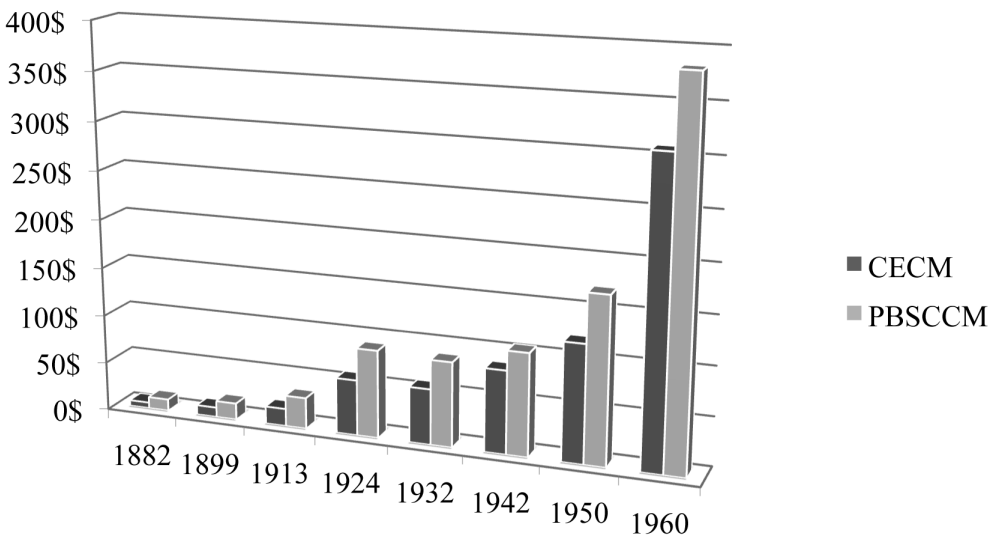

Sources: Rapports financiers. CECM. Archives de la Commission scolaire de Montréal. (ACSDM). Annual Report. PBSCCM. English Montreal School Board Archives (EMSBA). 


\section{Graphique 3}

Les sommes du Fonds d'éducation supérieure allouées aux collèges privés catholiques et aux écoles primaires supérieures et secondaires publiques (1930-1960) Années choisies

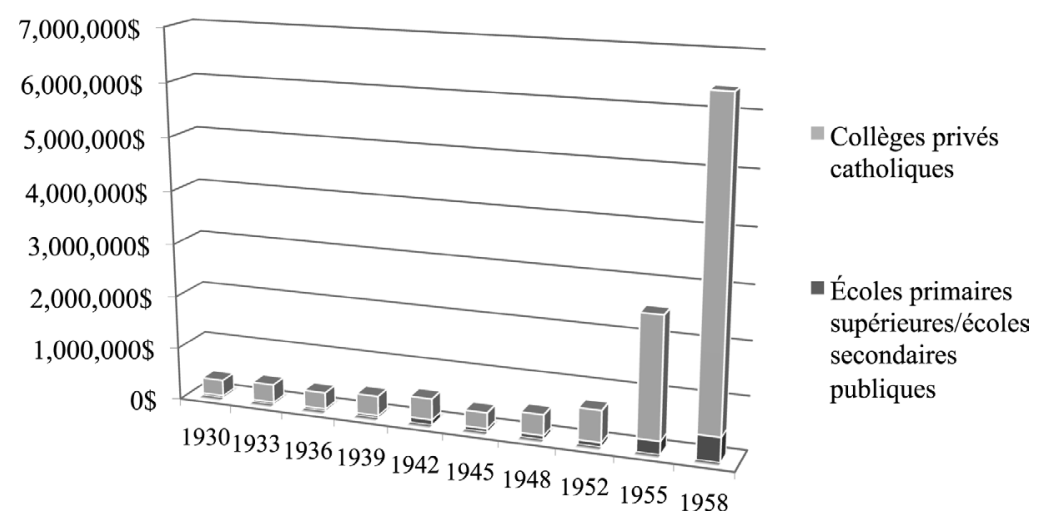

Sources: Rapports du surintendant de l'Instruction publique de la Province de Québec (RSIPPQ). 


\section{Tableau 1}

Taux d'évaluation de la propriété foncière par $100 \$$

à Toronto selon les listes de cotisation (1920-1960) (Années choisies)

\begin{tabular}{|c|c|c|}
\hline & TSSB & TBE \\
\hline 1920 & 1,20 & 0,80 \\
\hline 1921 & 1,15 & 0,70 \\
\hline 1923 & 1,15 & 0,70 \\
\hline 1940 & 1,15 & 0,75 \\
\hline 1953 & 1,35 & 0,95 \\
\hline 1955 & 1,40 & 1,30 \\
\hline 1956 & 1,70 & 1,60 \\
\hline
\end{tabular}

Sources : Minute Book (1920-1960). Toronto District Catholic School Board Archives. (TDCSBA). Minute Book (1920-1960). Toronto District School Board Archives. (TDSBA).

\section{Tableau 2}

Taux d'évaluation de la propriété foncière par $100 \$$

à Montréal selon les listes de cotisation (1920-1960) Années choisies

\begin{tabular}{|c|c|c|}
\hline & CECM & PBSCCM \\
\hline 1920 & 0,70 & 0,70 \\
\hline 1921 & 0,70 & 1,00 \\
\hline 1923 & 0,70 & 1,00 \\
\hline 1940 & 1,00 & 1,00 \\
\hline 1953 & 1,00 & 1,50 \\
\hline 1955 & 0,80 & 1,50 \\
\hline 1956 & 0,80 & 1,20 \\
\hline
\end{tabular}

Sources : Rapports financiers, CECM, 1920-1960, Rapports financiers. Services des finances, Archives de la Commission scolaire de Montréal (ACSDM). Annual Report, PSBGM, 1920-1960, English Montreal School Board Archives (EMSBA). 


\section{Notes}

1 Alison Prentice, The School Promoters, Education and Social Class in Mid-Nineteenth Century Upper Canada, Toronto, McClellan \& Stewart Inc., 1977, 47.

2 Egerton Ryerson, The Kind of Education which Canadian Youths Require and Hints to Them for Its Attainment, Victoria University Archives, 1841, 50 p. Cité dans Albert F. Fiorino, "The Moral Education of Egerton Ryerson's Idea of Education ", dans Neil MacDonald et Alf Chaiton (dir.), Egerton Ryerson and His Times, Toronto, Macmillan of Canada, 1978, 67.

3 J.M. Mangan et A. Davidson-Harden, "Ontario Schools in Social and Cultural Context ", dans J.M. Mangan (dir.), Social Foundations of Education Coursebook 2004-2005, London, Ontario, The University of Western Ontario, 2004, 1-2.

4 Voir R. D. Gidney, "Egerton Ryerson ", Dictionnaire biographique du Canada, 1881-1890, volume XI, Toronto et Sainte-Foy, University of Toronto, Université Laval, 1982, 783-795.

5 John S. Moir, Church and State in Canada West, Three Studies in the Relation of Denominationalism and Nationalism, 1841-1867, Toronto, University of Toronto Press, 1959, 148.

6 John S. Moir, "Toronto's Protestants and Their Perceptions of Their Roman Catholic Neighbours ", dans Brian P. Clark et Mark G. McGowan, Catholics at the Gathering Place, Historical Essays on the Archdiocese of Toronto, 1841-1991, Canadian Catholic Historical Association, Toronto, Dundurn Press Ltd, 1993, 313-327.

7 Murray W. Nicolson et John S. Moir, "Armand-François-Marie de Charbonnel», Dictionnaire biographique du Canada, volume XII, 1891-1900, University of Toronto et Université Laval, Québec, 1990, 198-202.

8 Charbonnel to the City Superintendent of Common Schools, 14 octobre 1850. Charbonnel Papers. Archives of the Roman Catholic Archdiocese of Toronto (ARCAT). Cité dans Walker, Catholic Education and Politics..., 95-96.

9 Cité dans Journal of Education, Upper Canada, vol. V, nº10 (octobre 1852), 145-148.

10 Egerton Ryerson, Report on a System of Public Elementary Instruction (1846), dans John George Hodgins, Documentary History of Education in Upper Canada, Ontario Department of Education vol. 6, Warwick Bros. \& Rutter Printers, 1894, 47-150.

11 John S. Moir, Church and State in Canada West..., 189.

12 Cité dans C. B. Sissons, Church and State in Canadian Education, Toronto, Ryerson Press, 1959, 20.

13 Voir Robert M. Stamp, The Historical Background to Separate Schools in Ontario, Toronto, Ministry of Colleges and Universities, 1985, 5-6 et Michael Power, A Promise Fulfilled, Highlights in the Political History of Catholic Separate Schools in Ontario, Toronto, Ontario Catholic School Trustee's Association, 2002, 525 p.

14 Minutes. Archives of Toronto Catholic District School Board (TCDSBA).

15 Voir Robert T. Dixon, We Remember, We Believe, A History of Toronto's Catholic Separate School Boards, 1841 to 1997, Toronto, Toronto Catholic District School Board, 2007, 181. Voir aussi les documents suivants, The Roman Catholic Separate Schools of Ontario, Brief Submitted to the Royal Commission on Education by the Ontario Catholic Education Council (1945), 1-12. Separate Schools, Documents Presented to the Royal Commission on Education in Ontario, The English Catholic Education Association of Ontario, Toronto, 1946, 1-79. Files of Dr. J. M. Bennett (c. 1919-1968). Box \# 4. TCDSBA.

16 Voir Mgr Neil McNeil, School Question of Ontario by the Archbishop of Toronto, Toronto, 1930, 28 p.

17 Mark G. McGowan, "The De-Greening of the Irish: Toronto Irish-Catholic Press, Imperialism and the Forging of A New Identity, 1887-1914 ", Historical Papers/ Communications historiques, vol. 24, $\mathrm{n}^{\circ} 1$ (1989), 118-145. 
18 Mark G. McGowan, The Waning of the Green, Catholics, the Irish and Identity in Toronto, 1887-1922, Montreal and Kingston, McGill-Queen's University Press, 1999, 118-148.

19 Reverend Neil McNeil, "A Need of the Day ", Separate School Chronicle, vol. 2, n³, décembre 1919, 1-2.

20 Voir Mgr, Neil McNeil, School Question of Ontario by the Archbishop of Toronto, Toronto, 1930, $28 \mathrm{p}$.

21 M. J. Quinn, Some Pertinent Facts, With Notes, Comments and Quotations for the Use of Those Who Desire to Understand and Discuss Publicly or Privately, the Situation of the Separate Schools of Ontario, Toronto, 1932, 22 p. Ontario School Tax Legislation, Speech By Hon. Arthur W. Roebuck, K.C. M. L. A., Attorney General of Ontario, Delivered in the Ontario Legislature, April 6th, 1936, 11 p. Files of Dr. J. M. Bennett (c. 1919-1968). Box \# 4. ATCDSB.

22 Toronto Board of Education, Minutes (1885-1962), Toronto Board of Education (TBEA) et Toronto District Catholic School Board, Minutes (1885-1962), TCDSBA. A Report of the Metropolitan Separate School Board to the Committee Appointed by his Honour the Lieutenant-Governor of Ontario, Order in Council-2 $2^{\text {nd }}$ day of may 1957. OC1513/57. Briefs/ Submissions to Government by MSSB. TCDSBA. Statement by the Honourable John Robarts, Prime Minister of Ontario, Concerning the Ontario Foundation Tax Plan, 1964, Delivered in the Ontario Legislature on Thursday, February 215t, 1963, 1-34. EDG000134. ARCAT.

23 Toronto and Suburban Separate School Board. Brief to Be Submitted to the Royal Commission of Education, 3. RG18-131. File: Brief 145, B249572. Archives publiques de l'Ontario (APO). Voir aussi Separate Schools, Documents presented to the Royal Commission on Education in Ontario, The English Catholic Education Association of Ontario, 8 novembre 1946, 6-8, Hope Commission (Henderson Papers). 1940s-1950s. TCDSBA.

24 English Catholic Education Association of Ontario. Public School Trustees Association of Ontario. Statement Relative to the Report of the Royal Commission on Education, March $7^{\text {th }}$, 1951, 1-4. EDHP0201 (j). ARCAT.

25 Voir Brief to Be Presented to the Government of Ontario by the Catholic Bishops of Ontario, Ontario Bishops Committee of Education, 5 septembre 1962. MSSB and Bishop Committee (1962). Henderson Papers. TCDSBA.

26 Toronto Board of Education, Minutes (1885-1962), ATBE et Toronto District Catholic School Board, Minutes (1885-1962), TCDSBA.

27 "Separate Schools: Should they get more of the tax dollar ? ", The Toronto Daily Star, 28 novembre 1962.

28 Voir "Separate school aid to be election issue Catholics warn Davis ", The Toronto Star, 21 juillet 1971. "Back to the religious wars ", The Globe and Mail, 22 juillet 1971. Statement of Roman Catholic Bishops of Ontario at Semi-Annual Meeting, 15 septembre 1971. Catholic Bishops' Statement (After Government refuses to compete), 1971. Henderson Papers. TCDSBA.

29 Pierre Carignan, "La religion dans les écoles publiques selon la loi de 1841 ", Thémis, La Revue juridique, vol. 17 (1982-1983), 19. Voir aussi, Jean-Pierre Charland, " Le réseau d'enseignement public bas-canadien, 1841-1867 : une institution de l'État libéral ", Revue d'histoire de l'Amérique française, vol. 40, n4 (printemps 1987), 507511 et 524-528. Jean-Pierre Charland, L'entreprise éducative, 1840-1900, Sainte-Foy, Presses de l'Université Laval (PUL), 2000, 60-61. Voir aussi Andrée Dufour, Tous à l'école. État, communautés rurales et scolarisation au Québec de 1826 à 1859, 1996, Montréal, Hurtubise-HMH, 91-121.

30 Mélanges religieux, 23 juillet 1840. Cité dans Carignan, «La religion dans les écoles publiques...", 38-39. 
31 Pierre Carignan, "L'établissement du système confessionnel d'enseignement sous le régime de l'Union ", Thémis, La Revue juridique, vol. 52 (1964), 272-274.

32 Rapport du surintendant de l'Éducation du Bas-Canada pour l'année 1855, 55. Rapport du surintendant de l'éducation du Bas-Canada pour l'année 1866, 46.

33 Louis-Philippe Audet, Histoire de l'enseignement au Québec, 1608-1971, tome 1, Montréal, Holt, Rinehart et Winston, 1971, 82-83.

34 Oscar Douglas Skelton, Life and Times of Sir Alexander Tilloch Galt, McClelland and Stewart Limited, Toronto, 182-185.

35 Cité dans Franklin Walker, Catholic Education and Politics in Upper Canada, Toronto, J. M. Dent \& Sons Ltd., 1954, 293.

36 "Meeting at Montreal and Formation of an Association for the Promotion and Protection of the Educational Interests of Protestants in Lower Canada », Journal of Education for Lower Canada, vol. VIII, n ${ }^{\circ}-10$ (septembre et octobre 1864), 151-153.

37 Voir aussi le discours prononcé par le principal de l'Université McGill, J. W. Dawson, en janvier 1864. J. W. Dawson, On Some Points in the History \& Prospects of Protestant Education in Lower Canada, A Lecture, Delivered by Principal Dawson, Before the Association of Teachers in Connection with the McGill Normal School, Dec. 1864, Montreal, 1864, 12-14.

38 Pierre Carignan, «La raison d'être de l'article 93 de la Loi constitutionnelle de 1867 à la lumière de la législation préexistante en matière d'éducation ", Thémis, La Revue Juridique, vol. 20, (1986), 442-449.

39 "Loi sur l'éducation ", Journal de l'éducation, vol. XIII, n 4 (avril 1869), 50-51.

40 Voir les travaux de Louis-Philippe, Audet, Histoire du Conseil de l'instruction publique de la province de Québec, 1856-1964, Montréal, Léméac, 1964, 346 p. et Ruby Heap, "Les relations Église-État dans le domaine de l'enseignement primaire public au Québec : 1867-1899", Société canadienne d'histoire de l'Église catholique, Sessions d'étude (1983), 183-199.

41 Le 16 janvier 1899, Livre des délibérations des commissaires (LDC), Archives de la Commission scolaire de Montréal (ACSDM).

42 Rapport financier du Bureau des commissaires d'écoles catholiques romaines de la Cité de Montréal pour l'exercice 1898-1899, Montréal, 1900, 5. Rapports financiers, Services des Finances, ACSDM.

43 Montreal Protestant Central School Board, Financial Statements (1925-1945). English Montreal School Board Archives (EMSBA).

44 Au sujet de la contestation de la loi de 1869 par les protestants, voir les études suivantes : Jean-Philippe Croteau et Robert Gagnon, "Les débats sur le partage de la taxe scolaire à Montréal (1869-1899) : enjeux et rivalités ", Revue d'histoire de l'éducation/Historical Studies in Education, vol. 20, $\mathrm{n}^{\circ} 1$ (printemps 2008), 32-68 et Jean-Philippe Croteau, "La question de la taxe scolaire à Montréal au XIX ${ }^{\mathrm{e}}$ siècle : un nouveau regard sur l'intégration sociale des juifs (1870-1903) ". Canadian Ethnic Studies/Études ethniques canadiennes, volume 41, no1-2 (2009), 1-28.

45 Dawson, On Some Points in the History \& Prospects..., 8-13.

46 "Meeting at Montreal and Formation of an Association for the Promotion and Protection of the Educational Interests of Protestants in Lower Canada ", Journal of Education for Lower Canada, VIII, n $9-10$ (septembre et octobre 1864), 151-153.

"Correspondance parlementaire de La Minerve », La Minerve, le 6 avril 1869.

47 "Correspondance parlementaire de La Minerve", La Minerve, le 6 avril 1869.

48 "The Convention ", The Educational Record of the Province of Quebec, vol. VII, n 11 , novembre 1887, 307.

49 Dans les années 1920 et 1930 , seules les classes de la $5^{\mathrm{e}}$ à la $8^{\mathrm{e}}$ année peuvent recevoir des subventions gouvernementales. Ce n'est qu'à partir de 1937 que les classes primaires supérieures $\left(9^{\mathrm{e}}, 10^{\mathrm{e}}, 11^{\mathrm{e}}\right.$ année) sont admissibles à recevoir des octrois du Fonds de l'éducation supérieure. 29 septembre 1937. Procès-verbaux du comité catholique (PVCC). 
50 Rapport du surintendant de l'Instruction publique de la province de Québec 1945-46 (RSIPPQ), 9.

51 Mémoire de l'Université Laval à la Commission royale d'enquête sur l'enseignement, juin $1962,86$.

52 Rapport du surintendant de l'Instruction publique de la province de Québec 1957-58 (RSIPPQ), 11 et Rapport du surintendant de l'Instruction publique de la province de Québec 1958-1959(RSIPPQ), 10.

53 Commission d'enquête sur la répartition des impôts municipaux et scolaires. Rapport sur les aspects financiers du problème scolaire, (1945-1946), 6 et 14. Taxes. Services des Finances. ACSDM.

54 Voir Robert Gagnon, Anglophones at the CECM, Montréal, CECM, 1996, 49-63 et Mélanie Lanouette, Penser l'éducation, dire sa culture, Les écoles catholiques anglaises au Québec, 1928-1964, Université Laval, thèse de doctorat (histoire), 2004, 277-295.

55 Statistiques des classes et des élèves par cours-30 septembre 1953. Services de la statistique. Services. ACSDM.

56 Voir le discours néo-nationaliste sur l'éducation dans Michael D. Behiels, Prelude to Quebec's Quiet Revolution, Liberalism versus Neonationalism 1945-1960, McGill-Queen's University Press, Montréal et Kingston, 1985, 148-184.

57 Arthur Tremblay, Contribution à l'étude des problèmes et des besoins de l'enseignement dans la province de Québec, Commission royale d'enquête sur les problèmes Constitutionnels, 1955, 162-163 et Mémoire de la Commission des Écoles catholiques de Montréal à la Commission Royale d'Enquête sur les problèmes constitutionnels, 14. Commission royale d'enquête sur l'éducation. ACSDM.

58 Rapport de la Commission royale d'enquête sur les problèmes constitutionnels, Volume III, Cinquième partie : Analyse des besoins et des recommandations, Tome I, La juridiction provinciale, Province de Québec, 1956, 186.

59 Protestant Board of School Commissioners of the City of Montreal, Annual Report (1872-1951). Montreal Protestant Central School Board, Annual Report (1945-1950). Protestant School Board of Greater Montreal, Annual Report (1951-1973). EMSBA.

60 "Trade Board Asks More Funds for Protestant Schools», The Montreal Daily Star, 18 février 1938 et "Schools in Need of Larger Grants ", The Montreal Daily Star, 14 mars 1938. "Trade Board Asks More Funds for Protestant Schools», The Montreal Daily Star, 18 février 1938.

61 Protestant Education in the Province of Quebec, Report of the Quebec Protestant Survey, 1938, 274-286; 321-323.

62 "Montreal Protestant Schools Send Autonomy Plea to Quebec ", The Montreal Star, 15 avril 1946.

63 "The School Question ", The Montreal Daily Star, 27 février 1947.

64 Rapport de la Commission royale d'enquête sur l'éducation, volume 4, chapitre I, par. 12-30 (1966).

65 Rapport de la Commission royale d'enquête sur l'éducation, volume 4, chapitre I, par. 26 (1966).

66 "Temporary Solution. Compromise on School Taxes", The Montreal Star, 6 juin 1965. Voir aussi "School Tax Problems ", The Gazette, 7 juin 1965.

67 Protestant School Board of Greater Montreal. Annual Report (1963-1970). EMSBA.

68 La Commission des écoles catholiques de Montréal. Rapport du trésorier (1962-1973) Rapports financiers. Service des Finances. Services. ACSDM.

69 "Un bulletin d'information à l'intention des commissions scolaires de leur personnel et des parents ", Le Bulletin du Conseil de l'Île de Montréal, Vol. I, nº 1, Mai 1973. Conseil scolaire de Montréal, Bulletin du Conseil scolaire de Montréal de l'île de Montréal (1973-1974). Système scolaire. Commissions scolaires locales. Commissions scolaires. Diverses généralités. ACSDM. 
70 Au sujet de la réforme du mode de financement scolaire à Montréal, voir Jean-Philippe Croteau, «La démocratisation du financement scolaire à Montréal : une révolution oubliée (1963-1973) ", Revue d'histoire de l'Amérique française (RHAF), vol. 62, no1 (été 2008), 5-34. 